\title{
Simultaneous acute Marchiafava-Bignami disease and posterior reversible encephalopathy syndrome: a case almost misdiagnosed
}

\author{
Liming Yang, Jing Liu, Yi Yin, Hui Yu \\ Affiliated Hospital of Guizhou Medical University, Guiyang 550004, China \\ Correspondence to: Hui Yu. Affiliated Hospital of Guizhou Medical University, Guiyang 550004, China. Email: 331693861@qq.com.
}

\begin{abstract}
Marchiafava-Bignami disease (MBD) is a rare neurological disease, and is characterized by callosal lesions consisting of necrosis and demyelination. Posterior reversible encephalopathy syndrome (PRES) is a clinical syndrome, and refers to a disorder with reversible subcortical vasogenic brain edema, predominantly involving the bilateral parieto-occipital regions. Never before had articles reported that the two diseases occurred simultaneously. We report a 49-year-old male alcoholic with both MBD and PRES who had a good prognosis after correct diagnosis and timely treatment.
\end{abstract}

Keywords: Marchiafava-bignami disease; posterior reversible encephalopathy; MRI; DWI

Submitted Nov 19, 2019. Accepted for publication Apr 16, 2020.

doi: 10.21037/qims-19-967

View this article at: http://dx.doi.org/10.21037/qims-19-967

\section{Introduction}

Marchiafava-Bignami disease (MBD) is a rare neurological disease that is often associated with chronic, heavy alcohol consumption and malnutrition. It is characterized by callosal lesions made up of necrosis and demyelination (1). Posterior reversible encephalopathy syndrome (PRES) refers to a disorder with reversible subcortical vasogenic brain edema caused by endothelial dysfunction which predominantly involves the bilateral parieto-occipital regions (2). Earlier studies reported that Marchiafava-Bignami disease could be accompanied by other comorbidities that share common pathogenesis in chronic alcohol abuse patients (3-5). To date, the co-occurrence of PRES and MBD has not been reported. Herein, we present a rare case of a patient with a history of chronic alcohol abuse who simultaneously suffered from MBD and PRES, which can provide a new diagnostic insight for radiologists who might encounter similar situations in clinical practice.

\section{Case presentation}

A 49-year-old man was sent to the emergency room with altered mental state and muscle strength reduction in the right limbs. The symptoms had started in the last four days and had gradually worsened. The patient was diagnosed with hypertension one month previously and had experienced a dizziness attack half a month before. He had consumed a daily average of $500 \mathrm{~mL}$ of liquor (Chinese spirits, $50 \%$ proof) for ten years. However, his blood and cerebrospinal fluid studies were within the normal limits.

Cranial CT presented a decreased density in the corpus callosum at the time of onset. FLAIR and T2WI imaging showed high-intensity signals and swelling in the genu, body, and splenium of the corpus callosum (Figure 1). Subcortical hyperintense lesions were also observed in the bilateral frontal, parietal, and occipital lobes as well as in the right basal ganglia, bilateral cerebellar hemisphere, and bilateral middle cerebellar peduncle (Figure 1A,B,C,D). Enhanced coronal images showed circular, linear enhancement in the body of the corpus callosum. DWI images demonstrated hyperintense lesions in the genu and the body of the corpus callosum, while multiple extracallosal lesions had low-to-equal intensity signals (Figure 1E,F,G,H). The susceptibility-weighted images (SWI) and the magnetic resonance angiography (MRA) of the patient appeared to be healthy.

Based on the clinical history and imaging features, the 

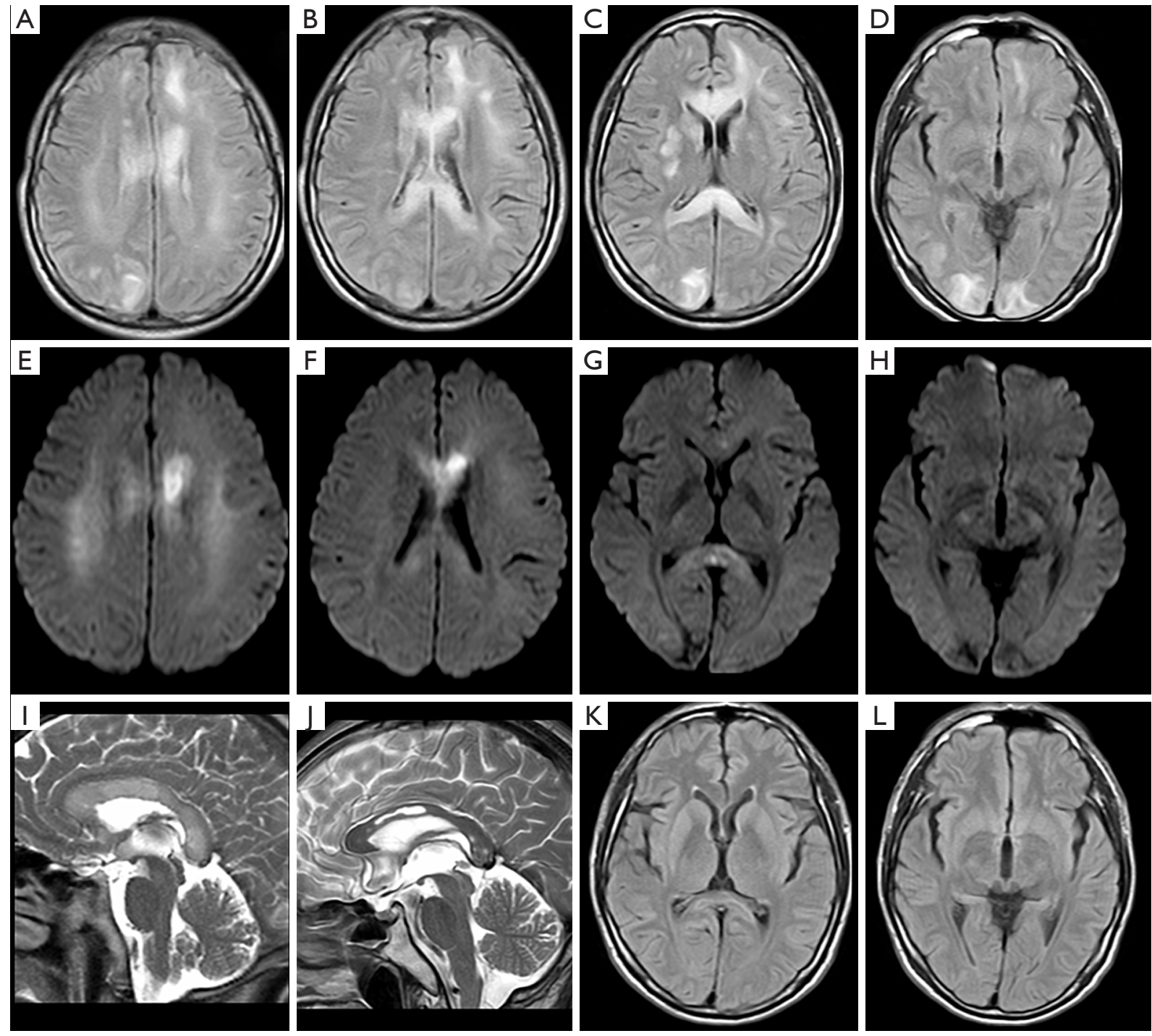

Figure 1 Initial MRI taken on admission. (A,B,C,D) FLAIR images show multiple hyperintense lesions in the frontoparietal-occipital subcortical regions, right basal ganglia, and the genu, body, and splenium of the corpus callosum exhibited hyperintense and swelling. (E,F,G,H) DWI images demonstrate hyperintensity in the genu and body of the corpus callosum. Multiple extracallosal lesions are low- to equi-intensity signals. (I) Sagittal T2WI images show high-intensity signals and swelling in the whole corpus callosum. Follow-up MRI examination. (J) Sagittal T2WI images show the 'sandwich sign' in the body of the corpus callosum, multiple cystic necroses is observed in the genu and splenium of the corpus callosum. (K,L) FLAIR images show slight atrophy of corpus callosum, and the disappearance of multiple extracallosal lesions.

patient was diagnosed as having MBD, and intravenous hyperalimentation was started with high-dose multivitamins, accompanied by continuous pumping of nitroglycerin. After three days of treatment, there was no visible improvement in the patient's condition; moreover, his blood pressure had risen to $171 / 99 \mathrm{mmHg}$. Through multidisciplinary consultation, we made a further diagnosis of MBD plus
PRES. Thus, with the use of amlodipine besylate to control symptoms of hypertension, the patient gradually regained consciousness, and the myodynamia of his right limbs improved. The follow-up MRI examination, which was performed 38 days after the initial examination, showed linear necrosis in the central layer of the body apart from the dorsal and ventral layers, as well as multiple cystic necroses 
in the genu and splenium of the corpus callosum (Figure 17). In contrast, the multiple lesions in the brain had almost disappeared (Figure 1K,L).

\section{Discussion}

MBD is a rare neurological disease often associated with chronic, heavy alcohol consumption and malnutrition, and is characterized by callosal lesions consisting of necrosis and demyelination (1). The typical MRI features of MBD are symmetric lesions of the corpus callosum. The impaired area has edematous changes with or without demyelination and appears as a high signal lesion on T2WI/FLAIR and DWI. After the acute stage, edema gradually subsides with high signal changes into a moderate or slightly low signal. If the lesions continuously progress to cause permanent myelin impairment or necrosis, the MRI shows atrophy and cystic transformation in the affected regions (3). Many factors, such as preeclampsia or eclampsia, renal failure, hypertensive crisis, sepsis, cytotoxic drugs, and autoimmune disorders, can cause PRES. The typical imaging manifestation of PRES is lesions with a high signal intensity in the parietal and occipital lobes on T2WI and FLAIR images, whereas the involvement of different regions, such as the frontal lobe, cerebellum, and basal ganglia, constitutes an "atypical" finding $(2,6)$

Because the clinical manifestations of these two diseases are diverse and nonspecific, their characteristic imaging manifestations and typical medical history are conducive to achieving a correct diagnosis. The acute onset of our patient was mainly manifested by altered mental state and reduced muscle strength in the right limbs, which might have easily caused misdiagnosis as stroke owing to the mimic clinical symptoms. At the outset, a diagnosis of MBD was established based on the patient's pivotal history of alcoholism and the characteristic MRI features of MBD, i.e., the corpus callosum lesions observed on images in multiple sequences, the enhancement patterns, and the well-known "sandwich sign" showed by the follow-up imaging examination during the changes of the lesions from the acute phase to the chronic phase (7). However, we failed to combine the hypertension history of the patient with the abnormal signals presented in bilateral frontal, parietal, and occipital lobes besides the corpus callosum lesions, which was consistent with the pathogenesis of typical vasogenic edema in PRES (6). Moreover, the follow-up images showed that the multiple lesions disappeared during the chronic phase, which also conformed with the imaging manifestations of PRES. Therefore, the patient's blood pressure was controlled using vitamins and antihypertensive drugs at the beginning of treatment. After giving the therapy based on the symptoms of both MBD and PRES, the satisfying outcome of the patient strongly supported our diagnoses of the concomitant diseases.

MBD can be divided into two types by combining clinical and neuroradiological manifestations (8). Type A is characterized by a history of acute and subacute onset, more severe clinical symptoms, and the whole corpus callosum involvement. Besides, lesions spreading to the residual regions of the brain and the poor outcome are common in patients of this type. Type B is characterized by an insidious clinical onset and might present milder symptoms than those of type A. The MRI images showed corpus callosum was partly involved and the spread of lesions to other regions was rare. Patients with this type usually have a favorable prognosis. Concerning our case, a diagnosis of type A MBD could be made based on both the history of acute onset and typical MRI findings. However, the cooccurrence of PRES complicated the clinical and imaging features. Thus, correct and prompt diagnosis will help neurologists to make proper treatment plans and implement them on patients in time.

This case reminds us that a comprehensive analysis of the patient's medical history, imaging manifestations, and treatment effects are of great significance for accurate diagnosis and favorable outcomes; this should be borne in mind in our future work.

\section{Acknowledgments}

Funding: None.

\section{Footnote}

Conflicts of Interest: All authors have completed the ICMJE uniform disclosure form (available at http://dx.doi. org/10.21037/qims-19-967). The authors have no conflicts of interest to declare.

Open Access Statement: This is an Open Access article distributed in accordance with the Creative Commons Attribution-NonCommercial-NoDerivs 4.0 International License (CC BY-NC-ND 4.0), which permits the noncommercial replication and distribution of the article with the strict proviso that no changes or edits are made and the original work is properly cited (including links to both the 
formal publication through the relevant DOI and the license). See: https://creativecommons.org/licenses/by-nc-nd/4.0/.

\section{References}

1. Shen YY, Zhou CG, Han N, Liang XM, Deng YQ. Clinical and neuroradiological features of 15 patients diagnosed with Marchiafava-Bignami disease. Chin Med J (Engl) 2019;132:1887-9.

2. Fittro K, Dizon R. Understanding posterior reversible encephalopathy syndrome. JAAPA 2018;31:31-4.

3. Hillbom M, Saloheimo P, Fujioka S, Wszolek ZK, Juvela S, Leone MA. Diagnosis and management of MarchiafavaBignami disease: a review of CT/MRI confirmed cases. J Neurol Neurosurg Psychiatry 2014;85:168-73.

4. Boloursaz S, Nekooei S, Seilanian Toosi F, RezaeiDalouei H, Davachi B, Kazemi S, Abbasi B. Marchiafava-

Cite this article as: Yang L, Liu J, Yin Y, Yu H. Simultaneous acute Marchiafava-Bignami disease and posterior reversible encephalopathy syndrome: a case almost misdiagnosed. Quant Imaging Med Surg 2020;10(6):1392-1395. doi: 10.21037/qims-19967
Bignami and Alcohol Related Acute Polyneuropathy: The Cooccurrence of Two Rare Entities. Case Rep Neurol Med 2016;2016:5848572.

5. Tsai CY, Huang PK, Huang P. Simultaneous acute Marchiafava-Bignami disease and central pontine myelinolysis: A case report of a challenging diagnosis. Medicine (Baltimore) 2018;97:e9878.

6. Tetsuka S, Ogawa T. Posterior reversible encephalopathy syndrome: A review with emphasis on neuroimaging characteristics. J Neurol Sci. 2019;404:72-9.

7. Kumar KS, Challam R, JN, Singh WJ. Marchiafavabignami disease: a case report. J Clin Diagn Res 2014;8:RD01-RD02.

8. Muccio CF, De Lipsis L, Belmonte R, Cerase A. Reversible MR Findings in Marchiafava-Bignami Disease. Case Rep Neurol Med 2019;2019:1951030. 